\title{
SHAPING HEALTHCARE THROUGH 3D PRINTING, INTEGRATING BIOINFORMATICS AND BIOMEDICAL RESEARCH
}

\author{
Dr. Mousumi Bhattacharjee James \\ Assistant Professor \\ New Horizon Institute of Technology and Management, Thane
}

\begin{abstract}
Bioprinting of Human organs as close replicas of the original are a result of interdisciplinary innovative research integrating Bioinformatics, Biotechnology and Biomedical sciences. The process of 3D and 4D manufacturing techniques have been devised over a period of time to manufacture products pertaining to multiple fields. This paper specifically discusses how this miraculous technology is evolving and is continuously changing lives. Various materials called bio-inks are used in this technology which involves a layer by layer addition of biomaterials with the help of robotic computer-aided technology resulting in biofabrication of functional 3D organ constructs. Biocompatible polymers are used as bio inks for the process. The lifestyle changes in mankind have led to various disorders increasing mortality and morbidity rates. In this complex chain of organ demand and supply where demand far exceeds supply, it is imperative to create a thrust on newer and advanced organ manufacturing technologies. This can be done by creating interest and impetus in this area of research by the world research organizations of importance. The high organ failure mortality rates across the globe have created an extreme shortage of donors. As discussed in this paper this emerging technology of organ bioprinting holds a great promise to mankind. The interdisciplinary nature of the subject needs focused deliberations of researchers from various disciplines of both medicine and technology to create a strong nexus resulting in even more incredible outcomes. This technology surely holds promise of better life for the future generations.
\end{abstract}

Keywords: Bioinformatics, Biotechnology, Biomaterials, Biomedical, Bioprinting, Biocompatible, Biofabrication, 3D Organ Printing.

\section{INTRODUCTION}

In this present age of interdisciplinary research, it is no longer feasible to limit innovative research to a particular field of study. When notable investigation and experimentation is in process; multidisciplinary fields of investigation and intellect needs to be integrated to arrive at an inference of substantial interest for the world community of scientists and innovative researchers. The study then being pursued further often results in positive benefits for the human race. In the present scenario of multidisciplinary research, the futuristic technology of multi-dimensional printing that is Three-dimensional (3D) and Four-dimensional (4D) printing has robustly emerged as the next generation of innovative and revolutionary technology, integrating various research areas, such as Bioinformatics, Chemistry, Life sciences, CAD, and materials science. 3D printing Techniques, the concept of which was laid in the 80's went on with further innovation to develop into 3D bio-printing. This was keeping in mind the benefit of technology for the human race. 3D technology enables the fabrication of high precision complex forms with the help of layer-by-layer addition of various materials, resulting in elements which can change shape or color, become electrically active, human tissue mimetic, or perform multiple functions. This technology of $3 \mathrm{D}$ and $4 \mathrm{D}$ printing has successfully paved the way for the production of dynamic multidimensional structures, intricate enough to mimic live tissues or organs. 3D bioprinting technology uses printing to combine various bio materials to fabricate biomedical parts that can copy tissue characteristics. 3Dimensional bio-printing is layer-by-layer deposition of bioinks to form structures which have been used successfully in the field of tissue engineering. This takes place with the help of bioinformatics for exact precision to form scaffolds of live tissue, for constructing patient specific scaffolds for various applications in tissue engineering. If the scientific imagination is permitted to explore the possibilities of research in this futuristic technology, and the benefits it could extend to mankind, we might as well infer that in the near future we could arrest the mortality and morbidity rates in multifold. To be precise organ banks would no longer have to wait for donors, the unending wait by organ failure patients, often resulting in untimely death before transplantation; could be now over. The artificial human organs created by this miraculous technology could be answering the prayers of multitudes of patients and their near and dear ones across the globe. A new dimension could be added to Healthcare research which could further lead to the investigative studies of diseases and drugs resulting in discovery of newer drug categories, vaccines, medical devices and the like for various medical conditions. 


\section{LITERATURE REVIEW}

The history of 3D printing goes back to the year 2003 when it was first introduced by Thomas Boland who patented the use of inkjet printing for cells for producing a cellular construct. This process made use of a modified spotting system for the deposition of cells into organized 3D matrices placed on a substrate $[1,2]$. Wilson and Boland, 2003 developed a cell printer which enabled them to place cells in positions that mimic their respective positions in organs [3]. An interesting study entitled Bioinformatics and 3D printing in Radiology Research: An Answer to Our Prayers discusses how this technology has altered the face of radio diagnosis and how bioinformatics has enabled researchers to print tissues and organs substitutes in actual human beings. The scientific community's wait for more than 100 years was finally over when their prayers were answered with Suzie et. al's development of tissue substitutes, further extremely realistic simulations are produced with physical and computational phantoms.[4]. A research by $\mathrm{Wu}$ et al concluded that $3 \mathrm{D}$ printing, also known as additive manufacturing technology, results in customized fabrication with the help of computer-aided design. With multifold advantages, this technology enables fabrication of tissue engineering scaffolds, with high precision. The appropriate scaffolds are designed, based on medical images obtained from computed tomography. This technology has been a boon to tissue engineering. [5].

An article by Xiaoming et al reviews how biopolymers are the excellent materials which have been judiciously applied in this technology; these materials have aroused great interest for potential applications in tissue engineering due to their ability to be shaped into any three-dimensional object referred from a digital model. The study revolves around recent advances in the development of biopolymer materials, including natural biopolymer based materials and synthetic biopolymer-based materials prepared using 3D printing technology, and the future challenges and applications of this technology are discussed[6]. A paper by Jian-Yuan Lee et al reviews the basic understanding of fundamentals of 3D printing processes and the recent development of novel 3D printing materials such as smart materials, ceramic materials, electronic materials, biomaterials and composites, which could become integral parts of a multiprocessing system[7]. Quoted in future outlook of a certain study by Meijuan Lei et al, threw light on how Bio-printing technology has obtained growing popularity and has made great progress with the pertinent techniques in tissue engineering, organ manufacturing and drug screening areas. Materials used are Biodegradable polymers are used as the cell printing accommodations, while stem cell techniques are especially helpful in organ manufacturing [8]. Various studies conducted across the globe prove how this futuristic technology has been a boon to the human race and is consistently transforming lives and hence holds a promising future. One such work by Wang, $\mathrm{X}$ et al states how hard tissues and organs, including the bones, teeth and cartilage, are the most extensively exploited and rapidly developed areas in regenerative medicine field. Further it was concluded that for hard organ regeneration, a series of new theories, strategies and protocols have been proposed. Some of the technologies have been applied in medical therapies with some success [9].

Toxicology testing by Hyun-Wook Kang et al, of new drugs has been traditionally applied on animal models, which apart from being expensive also implicates certain ethical considerations. This technology however allows the production of multiple types of living cells in combination utilizing an array of biomaterials for the construction of organoids which mimics organ or tissue on which studies are to be conducted [10]. A study by Dernowsek et al throws light on the nexus between applications of IT in biofabrication, how this technology can facilitate a shift from expensive in vitro and in vivo assays toward technologies which will allow exhaustive tests and optimization of human substitutes by means of computer biological systems [11]. A blog by Collins Francis quotes that "There's considerable excitement that 3D printing technology might one day allow scientists to produce fully functional replacement organs from one's own cells"[12]. A new dimension has been added via the medium of bone tissue 3D engineered constructs customized as per patientspecific needs as attractive bio-mimetic scaffolds to enhance bone cell and tissue growth and differentiation. The study by Brunello $\mathrm{G}$ et al, discusses the features of additive manufacturing technologies (3D printing, stereo-lithography, fused deposition modeling, and selective laser sintering) applied to fabricate bone tissue engineering scaffolds [13].

Shida Miao et al brought forth their work; four dimensional (4D) printing which first initiated in the year 2013 emerged as an intelligent technology with miraculous capacity for fabricating complex, stimuli-responsive 3D structures, furnishing integral requirements of tissue and organ engineering applications [14]. A review by Dilara et al presents a comprehensive survey of $3 \mathrm{D}$ and $4 \mathrm{D}$ printing methodologies, and discusses their advantage in tissue regeneration. It elaborates how Three-dimensional (3D) and Four-dimensional (4D) printing has turned out to be the next generation of fabrication techniques. It makes use of layer by layer deposition of materials to become bioactive and be used as intelligent materials for study and use in the field of tissue engineering and biotechnology [15]. A study conducted successfully by Pei Zhuang et al, in Nan yang Technological University Singapore discusses how, using the 3D Discovery's layer-by-layer UV curing system, researchers built thick cell-laden constructs with high shape fidelity and mechanical properties suitable for soft tissue engineering applications [16]. Zhuang $\mathrm{P}$ et al established that, this layer-by-layer ultraviolet assisted extrusion-based (UAE) Bio-printing may provide a novel strategy to develop soft tissue constructs with desirable structure integrity [17].

\section{BACKGROUND}


3D printing is not a new technology, In 1981, Hideo Kodama of Nagoya Municipal Industrial Research Institute invented two additive methods for fabricating threedimensional plastic models with photohardening thermoset polymer, where the UV exposure area is controlled by a mask pattern or a scanning fiber transmitter [18] [19]. The printing of human organs or organ bio-printing began in 1983 when Charles Hull invented stereo lithography. This printing relied on a laser to solidify a polymer material extruded from a nozzle. The instructions for the design, was decided by an engineer who would define the 3-D shape of an object in computer-aided design (CAD) software and then send the file to the printer. Hull and his colleagues developed the file format. On 8 August 1984, Charles W 'Chuck' Hull filed US patent number US4575330 A, for Apparatus for production of three-dimensional objects by stereo lithography that carried information about the object's surface geometry, represented as a set of triangular faces (Hull C. Apparatus for Production of Three-Dimensional Object by Stereo lithography). In the 30 years since Chuck Hull filed his patent on 3D printing the process of 'additive manufacture' (AM), has evolved and been used, continuously more and more applications have been found for AM. In the healthcare sector this technology has been extensively used, doctors and surgeons have been extensively using scan data to build 3D representations of patients' insides so much that the technologies inventor Chuck Hull has expressed surprise at the degree to which the medical industry had taken his invention [20].

\section{THE MATERIALS AND THE METHODS APPLIED}

Three dimensional printing has been commercially used in various fields of application in the industry and is continued to do so but as in this paper we are referring to bio-printing it has been felt quite apt to limit to three and four dimensional Bio-printing only which finds application for tissue engineering, prosthetics and organ transplantation. As per Singh et al, Three dimensional bioprinting is the utilization of printing techniques which combines cells, growth factors, and bio-materials resulting in biomedical parts that maximally imitate natural tissue characteristics [21]. It uses the layer by layer method to deposit materials known as bio inks to create tissue-like structures that are later used in medical and tissue engineering fields. This field of Bio-printing covers a broad range of bio materials. Hinton et al stated that, recently bioprinting finds use to print tissues and organs utilized for the research of drugs and pills [22]. Thomas, Daniel J expressed emerging innovations range from bio-printing of cells or extracellular matrix deposited into a 3D gel layer by layer to produce the desired tissue or organ. 3D bioprinting has begun to incorporate the printing of scaffolds [23]. Nakashima et al explained that the scaffolds are used to regenerate joints and ligaments, a boon to the field of orthopaedics [24]. As aptly mentioned in the present paper about interdisciplinary nature of this topic the following work describes Rodrigo A et al explained organ printing as an Information Technology which is supported by computer aided design for the fabrication of organs [25]. A variety of biomaterials are used in additive manufacturing to form the desired, complex-shaped products with different sizes and stiffness. Polymeric materials are generally preferred because of their easy process ability, biodegradability, biocompatibility, and low cost. Dilara Goksu Tamay et al stated that these materials are used in the form of filaments and powders, and as bio-inks for bioprinting. In this section, properties of materials used in $3 \mathrm{D}$ printing and bio-printing are discussed [26]. The process of Bio-printing of organs is a precision based, futuristic technology with a layer by layer addition of biomaterials with the help of robotic computer-aided technology resulting in biofabrication of functional 3D organ constructs. This technology utilizes self-assembling tissue spheroids according to predesigned digital model. Various soft-wares pertaining to Information technology and computer-aided design are integrated and eventually materialize in the transformation of virtual 3D bio-imaging about human tissue and organs which are reconstructed into living biological reality during 3D information bioprinting. Information technology facilitates design blueprints for bio-printing of human organs at the same time it also enables predictive computer simulation for both printing and post-printing processes [25].

Stimuli responsive polymers undergo physical or chemical changes when they are exposed to appropriate stimuli. The presence of certain functional groups along the polymer backbone creates this responsiveness and sensitivity to the change in state, such as charge or polarity. As the chemical structure changes, macroscopic level transformations take place, specifically changes in chain dimensions and size, secondary structure, solubility, degree of intermolecular association, sol-gel transition, and even chain breakage. Aguilar et al discussed an array of smart polymers in an extensive study [27]. A study by Karthik et al discusses the features of biocompatible materials used in this technology, that is, an ideal printing biomaterial must be biocompatible, conveniently printable with degradation rates, and one which can structurally mimic living tissue. The same study elaborates these materials can be categorized but the commonest of them are biopolymers which are biocompatible, biodegradable, and also leachable in body fluids [28]. This miraculous technology had the ability to produce fully functional replacements for impaired or injured tissues in patients. It can ably fabricate intricate and even miniature human-based tissue models, or phantom models, for pathology, diagnostics, and drug development studies. Bio-printing procedures or techniques include Cellular inkjet printing, Extrusion-based technologies, Soft Lithography, and Laser-induced forward transfer. It is imperative to mention that the whole process is not just advanced and innovative technology dependant but an enormous role is played by the nature of and biocompatibility of biomaterials selected. The accurate and definite integration of materials and method are responsible for supporting and aiding the cellular components during and after biofabrication. The wide array of biocompatible 
materials, include, curable synthetic polymers, synthetic gels, and naturally derived hydro gels. Skardal et al expressed the integration of bio-printing technologies with the help of exact biomaterials leads to bio-printed constructs with assured practical application in the field of medicine [29]. An extensive study by Natalja E et al, discussed that, once scaffolding is done the printed tissue regenerates, the mechanism of which has been comprehensively discussed [30]. The study which is in context of engineered bone grafts, discusses the united action of bone-forming cells, matrix and bioactive stimuli which then actuates the resultant performance of the implant. It is presently considered that the well-built 3D constructs comprise of the biological elements that restate or regenerate original bone tissue structure for bone formation once implanted. This is how this unbelievably wondrous technology of Bio-printing of organs is able to add a dynamic and positive dimension in the field of components pertaining to bone formation and also addresses issues, such as graft porosity and vascularization. Bone printing is perceived as a great blessing, it makes use of accurate prototyping technology for producing scaffolds of the desired shape and internal structure at the same time it consists of multiple living cell types incorporated which in turn can form the bone tissue once it is implanted. Classically Bio-printing is a $3 \mathrm{D}$ fabrication technique which can print complex tissue constructs using hydrogels loaded with cells to print. Mandrycky et al stated that this technology generates a variety of transplantable soft tissues, including skin, bone and cartilage [31].

\section{BIOPRINTING ANSWERING PRAYERS AND TRANSFORMING LIVES}

3D printing is indeed a medical marvel which offers everything from affordable prosthetics to replacement of certain body parts. This incredible technology is changing lives for the better and making us believe in the world of medical marvels. From tailor made heart sensors to heart valves, blood vessels, flexible bone implants, there are many such incredibly amazing bio-printed organs in use. Stem cell research advancements have made it possible to load living cells into a capable machine to 3D print tissues, organs and more to be used effectively inside the human body. The world of prosthetics has been gifted with multiple marvels as prosthetic nose, ears, arms; feet etc. research is on for vital organ cell prototyping. Statistical data by Allemani, $\mathrm{C}$ et al substantiates that, there were 25.7 million adults and 75,000 children suffering from cancer of stomach, liver, breast, lung, and so on during 1995-2009 [32] Dalal, A.R quoted that there were 117,040 patients that needed organ transplantation, but only 28,053 suitable organs available in the USA in 2013 [33]

Data such as these and many more suggest that organ transplantation is an imperative medical need of patients across the globe. Promising ongoing research in institutions around the globe are being done on the development of 3D-bioprinted organs such as the livers and kidneys with integrated vasculature, in order for the tissue to be able to thrive once it has been transplanted. Since Hull patented this technology, it has continuously evolved, but nevertheless much more needs to be achieved in the field of biomedical engineering to provide some solutions. With the advent of this wondrous technology the possibilities of development of tissues or organ grafts from cells might be within the reach. 3D bio-printers have been able to create biologically functional tissues by dispensing layer after layer of bio-ink and bio-gel made to mature with the proper environment producing a functional tissue copy with normal metabolic activity. Today, the technology reaching peaks has enabled 3D-bioprinted bladders, tracheal grafts, bone, and cartilage which are developed and implanted in animal models and humans. Organ bio-printing is indeed an answer or solution to check mortality and morbidity rates across the world. Patients on the waiting list for transplantation of kidneys, pancreas, liver, or heart are on a miserable trail and a long journey of hopeless wait. Tens of thousands of people find themselves in this dire situation every year. Only a fortunate few have their prayers answered, the rest fall short of breath till their wait for a donor is over. Eventually, scientists look forward to print patient-specific organs that can be transplanted safely into the human body. Though the debate is on about the ethical aspect of organ printing where patients will no longer have to wait for donors and their organ prototypes can be just printed in a jiffy and transplanted. This innovative Bio-printing technology could bring in a future where organs for transplantation could not only be printed economically, but also to the exact anatomical specifications of each individual patient. While extensive research still needs to be done in this arena, but nevertheless one can easily perceive that the sky is the limit for this innovative technology which is fast changing the lives of the human race for the better.

\section{CONCLUSION}

In this byzantine chain of organ demand and supply where demand far exceeds supply it is imperative to create a thrust on newer and advanced organ manufacturing technologies. This can be done by creating interest and impetus in this area of research by the world research organizations of importance. The, increasing lifestyle changes leading to high organ failure mortality rates across the globe has indeed created an extreme shortage of donors. As discussed in this paper this emerging technology of organ bio-printing holds a great promise to mankind. The interdisciplinary nature of the subject needs focused deliberations of researchers from various disciplines of both medicine and technology to create a strong nexus resulting in even more incredible outcomes. How encouraging it is to imagine about the promises that the world of medical marvels of which this technology of bioprinting is just a part, surely holds of better life for the future generations. 


\section{ACKNOWLEGEMENT}

I would like to take this opportunity to extend my gratitude to New Horizon Institute of Technology and Management, Thane, under Mumbai University for providing the platform for my work.

\section{REFERENCES}

[1] Boland, T, (2003)_Patent US7051654: Ink-jet printing of viable cells".

[2] Auger, François A.; Gibot, Laure; Lacroix, Dan (2013). The Pivotal Role of Vascularization in Tissue Engineering, Annual Review of Biomedical Engineering. 15: 177-200

[3] Boland Thomas, Mironov Vladimir, Gutowska Anna, Roth Elisabeth. A., and Markwald Roger R., Cell and Organ Printing: Fusion of Cell Aggregates in ThreeDimensional Gels, ( 2003), (C) 2003 WILEY-LISS, INC.

[4] Susie M O, Sergio Augusto L D S, Lidia Vasconcellos D S.( 2018) Bioinformatics and 3D Printing in Radiology Research: An Answer to Our Prayers. BJSTR, MS.ID.001712. DOI: 10.2671/BJSTR.2018.08.001712.

[5] Wu, GH. \& Hsu, S. (2015) Review: Polymeric-Based 3D Printing for Tissue Engineering, J. Med. Biol. Eng. 35: 285 .

[6] XiaomingLi, Rongrong Cui, Lianwen Sun, Katerina, Aifantis E., Yubo Fan, Qingling Feng, Fuzhai Cui, and Fumio Watari, (2014) 3D-Printed Biopolymers for Tissue Engineering Application, International Journal of Polymer Science Volume Article ID 829145, 13 pages

[7] Jian-Yuan Lee, Jia An Chee, Kai Chua (2017) Fundamentals and applications of 3D printing for novel materials, Applied Materials Today, Volume 7, June, Pages 120-133

[8] Meijuan Lei , Xiaohong Wang (2016) Biodegradable Polymers and Stem Cells for Bioprinting, Molecules, 21(5), 539

[9] Wang, X.; Ao, Q.; Tian, X.; Fan, J.; Wei, Y.; Hou, W.; Tong, H.; Bai, S.( 2016) 3D Bioprinting Technologies for Hard Tissue and Organ Engineering; Materials , 9, 802.

[10] Hyun-WookKang Anthony Atala James J.Yoo ( 2015) Essentials of 3D Biofabrication and Translation, Elsevier Inc, Pages 179-186

[11] Dernowsek, Janaína, Rezende Rodrigo , Silva, Jorge. (2017). The role of information technology in the future of 3D biofabrication. Journal of 3D Printing in Medicine. 1. 63-74.

[12] Collins Francis (2019) NIH Director's blog, Progress Toward 3D Printed Human Organs.
[13] Brunello G, Sivolella S , Meneghello R , Ferroni L , Gardin C , Piattelli A , Zavan B , Bressan E (2016) Powder-based 3D printing for bone tissue engineering. Biotechnol Adv. Sep-Oct; 34(5):740-753.

[14] Shida Miao1, Nathan Castro, Margaret Nowicki, Lang Xia, Haitao Cui, Xuan Zhou, Wei Zhu, Se-jun Lee, Kausik Sarkar, Giovanni Vozzi, Yasuhiko Tabata, John Fisher, Lijie Grace Zhang, (2017) 4D printing of polymeric materials for tissue and organ regeneration, Materials Today 20(10):577-591.

[15] Dilara Goksu Tamay, Tugba Dursun Usal, Ayse Selcen Alagoz, Deniz Yucel, Nesrin Hasirci , Vasif Hasirci ( 2019) 3D and 4D Printing of Polymers for Tissue Engineering Applications, Frontiers in Bioengineering and Biotechnology, Vol 7, Article 164.

[16] Pei Zhuang, Wei Long Ng, Jia An, Chee Kai Chua, Lay Poh Tan (2019) Nan yang Technological University Singapore, Layer-By-Layer Ultraviolet Assisted ExtrusionBased (UAE) Bioprinting Of Hydrogel Constructs With High Aspect Ratio For Soft Tissue Engineering Applications, Posted in Partners achievements.

[17] Zhuang P, Ng WL, An J, Chua CK, Tan LP (2019) Layer-by-layer ultraviolet assisted extrusion-based (UAE) bio printing of hydro gel constructs with high aspect ratio for soft tissue engineering applications. PLoS ONE 14(6): $\mathrm{e} 0216776$.

[18] Hideo Kodama, (1981) A Scheme for ThreeDimensional Display by Automatic Fabrication of ThreeDimensional Model, IEICE Transactions on Electronics (Japanese Edition), vol. J64-C, No. 4, pp. 237-41.

[19] Hideo Kodama, (1981) Automatic method for fabricating a three-dimensional plastic model with photohardening polymer, Review of Scientific Instruments, Vol. 52, No. 11, pp. 1770-73.

[20] Whitaker Matthew,( 2014) The history of 3D printing in healthcare, RCS, The Bulletin, Volume: 96 Issue: 7, pp.228-229.

[21] Singh, Deepti, Thomas, Daniel (2018). Advances in medical polymer technology towards the panacea of complex 3D tissue and organ manufacture, American Journal of Surgery. 217 (4): 807-808.

[22] Hinton TJ, Jallerat Q, Palchesko RN, Park JH, Grodzicki MS, Shue HJ, Ramadan MH, Hudson AR, Feinberg AW (2015). Three-dimensional printing of complex biological structures by free form reversible embedding of suspended hydrogels. Science Advances.1 (9)

[23] Thomas, Daniel J. (2016) Could 3D bioprinted tissues offer future hope for microtia treatment? International Journal of Surgery. 32: 43-44.

[24] Nakashima, Yasuharu; Okazak, Ken; Nakayama, Koichiet; Okada, Seiji; Mizu-uchi, Hideki (2017). Bone 
and Joint Diseases in Present and Future, Fukuoka Igaku Zasshi Hukuoka Acta Medica. 108 (1)

[25] Rodrigo A. Rezende; Vladimir Kasyanov; Vladimir Mironov; Jorge Vicente; Lopes da Silva, (2015) Organ Printing as an Information Technology, Procedia Engineering, Volume 110, Pages 151-158.

[26] Dilara Goksu Tamay et al (2019) 3D and 4D Printing of Polymers for Tissue Engineering Applications, Front Bioeng Biotechnol. 7: 164.

[27] Aguilar and San Román (2014 ) Smart Polymers and their Applications, Woodhead Publishing, ISBN: 9780857096951.

[28] Karthik Tappa and Udayabhanu Jammalamadaka (2018) Novel Biomaterials Used in Medical 3D Printing Techniques, J Funct Biomater , 9(1): 17.doi: $10.3390 /$ jfb9010017

[29] Skardal, A. \& Atala, A. (2015) Biomaterials for Integration with 3-D Bioprinting, Ann Biomed Eng 43: 730 .

[30] Natalja E. Fedorovich; Jacqueline Alblas; Wim E. Hennink F; Cumhur Oner Wouter J. A. Dhert (2011) Organ printing: the future of bone regeneration, Trends in Biotechnology, Volume 29, Issue 12, Pages 601-606.

[31] Mandrycky et al., 2016.

[32] Allemani, C.; Weir, H.K.; Carreira, H.; Harewood, R.; Spika, D.; Wang, X.-S.; Bannon, F.; Ahn, J.V.; Johnson, C.J.; Bonaventure, A.; et al. (2015) Global surveillance of cancer survival 1995-2009: (CONCORD-2). Lancet, 385, 977-1010.

[33] Dalal, A.R. (2015) Philosophy of organ donation: Review of ethical facets. World J. Transplant. 24, 44-51. 